\title{
Neuroblastom of the Bone Marrow in Young Adult Male Without Identifiable Primary
}

ISSN: 2637-773X

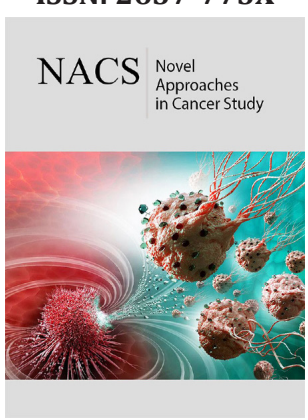

*Corresponding author: Basil Kadhim Abdullah, Consultant subspecialty pediatric heamato-oncology, Sulemania, Iraq

Submission: 㥁: October 22, 2020

Published: 眥December 15, 2020

Volume 5 - Issue 4

How to cite this article: Basil Kadhim Abdullah*, Zainab Basil Kadhim, Ghada Basil Kadhim, Zena Jameeel Ahmed. Neuroblastom of the Bone Marrow in Young Adult Male Without Identifiable Primary. Nov Appro in Can Study. 5(4). NACS.000619. 2020. DOI: 10.31031/NACS.2020.05.000619

Copyright@Basil Kadhim Abdullah, This article is distributed under the terms of the Creative Commons Attribution 4.0 International License, which permits unrestricted use and redistribution provided that the original author and source are credited.

\author{
Basil Kadhim Abdullah' ${ }^{1 *}$, Zainab Basil Kadhim², Ghada Basil Kadhim ${ }^{3}$ and \\ Zena Jameeel Ahmed ${ }^{4}$ \\ ${ }^{1}$ Consultant subspecialty pediatric heamato-oncology, Iraq \\ ${ }^{2}$ Medical student, School of medicine, Iraq \\ ${ }^{3}$ Sixth stage student, freshta secondary School, Iraq \\ ${ }^{4}$ Jwahery primary school, Iraq
}

\begin{abstract}
Neuroblastoma is the most common malignancy in infancy and the third most common cancer in pediatric, following the acute leukemia and central nervous system tumor, constituting approximately $7-10 \%$ of pediatric malignancy. The majority of the patients (approximately $90 \%$ ) diagnosed before the age of 5 years, with peak incidence at the age of two years. However, the incidence of neuroblastoma decreasing with age and less than $5 \%$ from all cases diagnosed after the age of 10 years, its occurrence is rare during adulthood (one per 10 million cancer diagnoses per year). In addition, neuroblastoma is very rarely presented with Bone marrow involvement without identifiable primary site. We reported a young adult male patient with advanced neuroblastoma at the age of 21 with solely Bone marrow involvement without radiological identifiable primary tumor site who was died before starting him on chemotherapy treatment because of severe febrile neutropenia.
\end{abstract}

Keywords: Neuroblastoma; Young adult; Bone marrow involvement; Primary tumor; MYCN amplification Abbreviations: g: gram; dl: deciliter; ml: milliliter; PT: Prothrobine Time; PTT: Partial Thromboblastine; CD: Cluster of Differentiation; CT: Computerized Tomography; mg: milligram; VMA: Vanillyl Mandelic Acid; HVA: Homo Vanillic Acid (HVA)

\section{Introduction}

Neuroblastoma arises from primitive sympathetic neural cells primarily in the adrenal medulla and also in the paraspinal sympathetic ganglia in the neck, chest, abdomen, or pelvis [1]. Although neuroblastoma currently represents $7 \%$ of all childhood malignancies or roughly 1 case per 100,000 children per year, only 1 case per 10 million adults per year is diagnosed in adulthood [2-4]. Because of the rarity of adult neuroblastoma, staging systems and risk assessment tools have been developed using primarily pediatric data [5]. Clinically relevant pediatric factors that influence survival in children include stage, age, histology, tumor grade, MYCN oncogene status, chromosome 11q status, and DNA ploidy. These factors are currently part of the international neuroblastoma risk assessment system [6]. Because of the rarity of this tumor in adult patients (over 20 years old) We reported an adult male patient pancytopenia who was diagnosed as a bone marrow involved MYCN positive neuroblastoma without any radiological evident primary tumor site who was unfortunately died before directed antitumor therapy because of the rapid progressive course of febrile neutropenia.

\section{Case Report}

A 21-year-old young adult male was referred to the hematology department because of pancytopenia for assessment and treatment. The patient was well until he suddenly developed intermittent fever and anorexia for the last 5 weeks which was temporarily responding to antipyretic, he received several courses of antibiotic treatment without any objective response, the fever was neither associated with rigor nor sweeting. He was fully vaccinated with no consanguinity of parents. Medical and surgical histories were uneventful, family 
history was negative with no similar condition. On examination, he was febrile, ill looking, pallor, ecchymosis on both legs, neither hepatomegaly nor lymphadenopathy. Laboratory investigations reveal pancytopenia in complete blood picture with Hemoglobin value of $8.4 \mathrm{~g} / \mathrm{dl}$, the platelet count was $19,000 / \mathrm{ml}$, and a leukocyte count of $2,300 / \mathrm{ml}$ with $54 \%$ neutrophils, $45 \%$ lymphocytes, $1 \%$ monocytes, $0.5 \%$ basophils and $0 \%$ eosinophil, Blood film reveals normochromic normocytic anemia with neither immature nor abnormal cell, erythrocyte sedimentation rate $97 \mathrm{~mm}$ in $1 \mathrm{~h}$.

Other laboratory investigations including liver function tests, renal function tests, serum electrolytes (potassium, sodium, calcium, phosphorus); serum uric acid and coagulation profile (PT and PTT), all were normal. Bone marrow aspirate (BMA) morphologically revealed that normal hemopoietic cells were almost entirely (more than 90\%) replaced with small blue round neoplastic cells. These were mainly cells with a high nucleocytoplasmic ratio and moderately basophilic cytoplasm; tumor cells appear arranged in a rosette (Figure 1A \& 1B).Bone marrow trephine biopsy (BMB) confirmed the diagnoses of poorly differentiated neuroblastoma by morphology that showed infiltration with non-hematological malignant small round cell (Figure 2), and immunohistochemistry revealed positive staining for synaptophysin, Neuron Specific Enolase (NSE), CD56, and neuronal marker PGP9.5 (Figure 3). Real time polymers chain reaction (PCR) analysis for the tumor cells was positive. Radiological evaluations including abdominal ultrasound, chest $x$-ray, C.T. scan of chest and abdomen all were normal and failed to find an apparent primary site. 24 hours urine vanillylmandelic acid (VMA) was elevated with a value of $53 \mathrm{mg} / 24$ hours (normally less than 9mg/24 hours); 24 hours urine homovanillic Acid (HVA) was also elevated with a value of $61 \mathrm{mg} /$ day (normal level of less than $15 \mathrm{mg} /$ day). Based on the bone marrow morphology, immunohistochemistry staining and elevated 24 hours urine for both VMA a HVA the diagnosis of stage IV neuroblastoma was confirmed. The patient clinical condition was critical because of febrile neutropenia and blood culture was positive for pseudomonas aeruginosa which was sensitive to meropenem, he deteriorated rapidly and unfortunately died because of severe sepsis before starting chemotherapy or other-directed therapy for his underlying malignancy. His critical, rapidly deteriorated clinical condition did not allow us to do neither Positron emission tomography (PET), metaiodobenzylguanidine (MIBG) nor Bone scan.

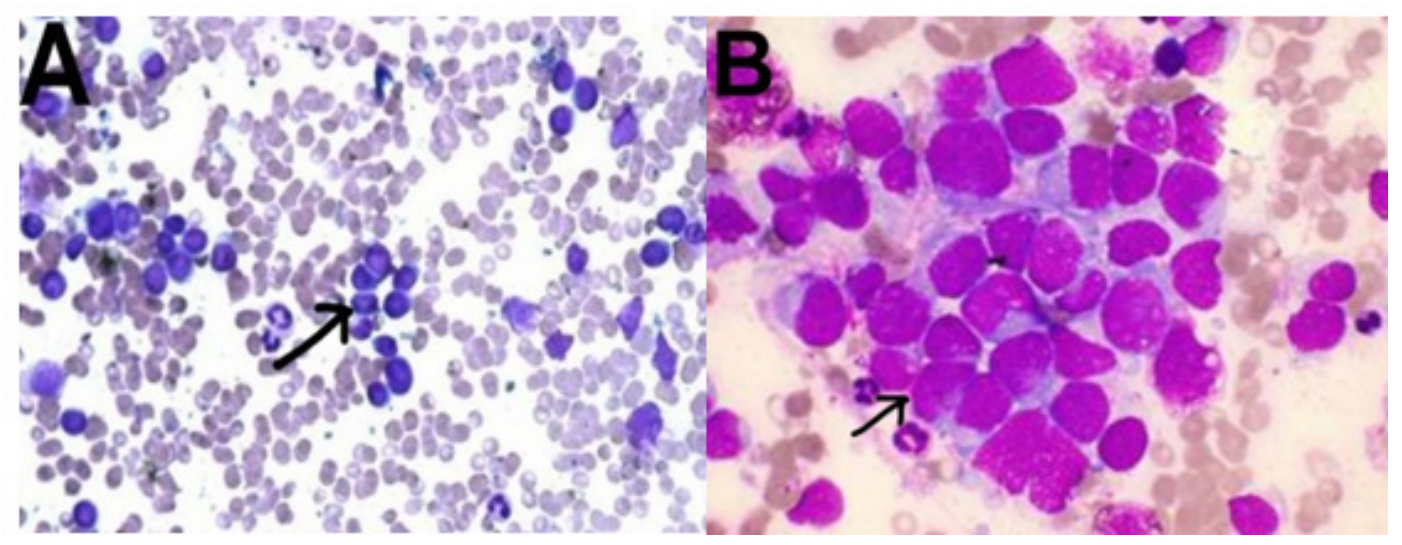

Figure 1: BMA shows normal hemopoietic cells replaced by small blue round neoplastic cells; tumor cells appear arranged in a rosette (black arrow) by Giemsa Stain.

A. (original magnification $200 \mathrm{X}$ ) and B. (original magnification 1000X).

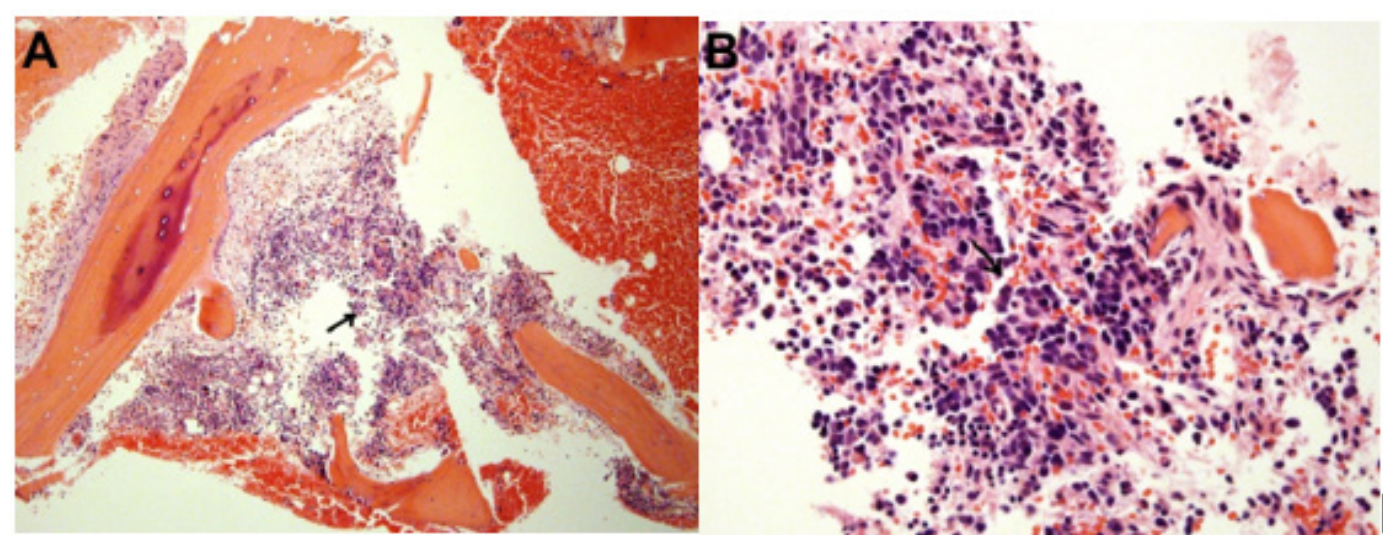

Figure 2: Bone marrow trephine biopsy shows infiltration with non-hematological small round cells malignancy (black arrow).

A. (H\&E, X100) and B.(H\&E, X400). 

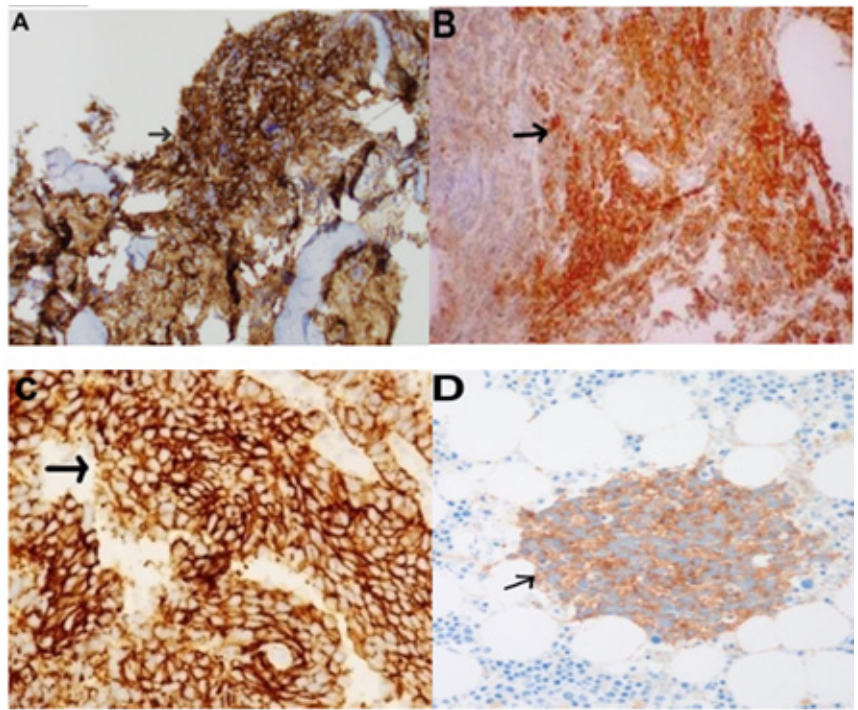

Figure 3: Bone marrow trephine biopsy immunohistochimestry reveals positive staining (black arrow) for synaptophysin.

A. Neuron Specific Enolase (NSE)

B. CD56

C. and pgp9.5

D. (original magnification 200X).

\section{Discussion}

Neuroblastoma is the most commonly occurring extracranial solid tumor diagnosed in the pediatric population [7]. Ninety percent of diagnoses occur in patients less than 4 years of age $[8,9]$. Furthermore, outcomes in adults are significantly worse than those in children. However, this diagnosis is extremely rare in adults, with less than 100 cases reported in the literature; moreover, adults with metastatic neuroblastoma typically have a very poor prognosis [10]. Disease presentation is often similar in adults and children, though several differences have been described. Disease progression is often more indolent in adults compared with children. A significant minority has a slowly progressive disease or recurrent relapses over a period of years. Tumors are of the sympathetic nerve chain and thus locations of primary tumors occur similarly in adults and children [11]. Notably, MYCN amplification is common in the highrisk pediatric population but appears very rarely in adults [12]. We reported this adult male with neuroblastoma for two reasons; first because of the rarity of neuroblatoma in adult (defined as older than 20 years) with less than 100 cases reported in the literature) [10]; and second because of this disease occasionally presented with solely bone marrow infiltration without identifiable primary site, in a series of 25 adult/adolescent patients, $16 \%$ had disease occurring in the pelvis, while $68 \%$ arose in the retroperitoneum or adrenal glands [12].

Our case report, we presented 21 years old young adult male presented with fever and pancytopenia, no hepatosplenomegaly, neither lymphadenopathy nor palpable mass, complete blood picture and blood film showed anemia, thrombocytopenia and neutropenia without any abnormal immature cells. BMA showed infiltration by malignant small blue round cells which replaced almost all hemopoietic components, immunohistochemical staining of the bone marrow trephine biopsy together with elevated tumor marker (24-hour urine VMA and HVA) confirmed the diagnosis of neuroblastoma which is categorized according to the international neuroblastoma staging system (INSS) as stage 4 (due to the bone marrow involvement).

Radiological evaluation by abdominal ultrasound, chest x-ray, CT scan of chest and abdomen; all failed to identify an apparent primary site. Unfortunately, the patient died because of severe sepsis due to febrile neutropenia before further radiological assessments by Positron emission tomography (PET), metaiodobenzylguanidine (MIBG) and Bone scan.

\section{Conclusion}

This case represents the presentation of a common disease in an uncommon patient population with uncommon presenting scenario. Due to the rarity of the neuroblastoma in adult and due to the fact that pancytopenia without primary mass; The majority of adult hematologists and oncologists will not put neuroblastoma in the differential diagnosis of neuroblastoma in an adult patient with pancytopenia; however, this diagnosis should at least be considered in certain cases. Neuroblastoma should be considered particularly in patients of adolescent age and possible cause of pancytopenia even in adults. Furthermore, due to the rarity of these diseases, diagnostic evaluation by an experienced pathologist is warranted to distinguish between neuroendocrine tumors and the rare case of adult neuroblastoma. Due to the rapidly progressive course of our patient and due to the MYCN amplification in our patient; we also concluded that neuroblastoma in adult patients is possible to behave more aggressive in adult patients than in pediatric patients. 


\section{Consent}

Written informed consent was obtained from patient's parent for this case report.

\section{Acknowledgment}

We gratefully acknowledge both patient and his family for allowing us to publish their case report.

\section{Authors' Contributions}

Consultant Dr. Basil Kadhim Abdallah Al odda has been involved in clinical diagnostic evaluations and management.

\section{Conflict of Interest}

The authors declare that they have no conflict of interests.

\section{Ethical Consideration}

Ethical approval for this case report was obtained from NCI ethical committee. Because of the retrospective nature of this case report, the ethical committee waived the requirement of informed consent.

\section{References}

1. Brodeur GM (2003) Neuroblastoma: Biological insights into a clinical enigma. Nature Reviews Cancer 3(3): 203-216.

2. Siegel R, Naishadham D, Jemal A (2012) Cancer statistics, 2012. CA: A Cancer Journal for Clinicians 62(1): 10-29.

3. Hsieh MH, Meng MV, Walsh TJ, Matthay KK, Baskin LS (2009) Increasing incidence of neuroblastoma and potentially higher associated mortality of children from nonmetropolitan areas: Analysis of the surveillance, epidemiology, and end results database. Journal of Pediatric Hematology/Oncology 31(12): 942-946.

4. Esiashvili N, Goodman M, Ward K, Marcus RB, Johnstone PA (2007) Neuroblastoma in adults: Incidence and survival analysis based on SEER data. Pediatric Blood and Cancer 49(1): 41-46.

5. Monclair T, Brodeur GM, Ambros PF, Brisse HJ, Giovanni, et al. (2009) The international neuroblastoma risk group (INRG) staging system: An INRG task force report. Journal of Clinical Oncology 27(2): 298-303.

6. Cohn SL, Pearson AD, London WB, Tom M, Ambors PF, et al. (2009) The international neuroblastoma risk group (INRG) classification system: An INRG task force report. Journal of Clinical Oncology 27(2): 289-297.

7. Castleberry RP (1997) Neuroblastoma. European Journal of Cancer 33(9): 1430-1437.

8. Maris JM (2010) Recent advances in neuroblastoma. The New England Journal of Medicine 362(23): 2202-2211.

9. Hiyama E, Iehara T, Sugimoto T, Masahiro F, Yutaka H, et al. (2008) Effectiveness of screening for neuroblastoma at 6 months of age: A retrospective population-based cohort study. The Lancet 371(9619): 1173-1180.

10. Franks M, Bollen A, Seeger RC, Stram DO, Matthay KK (1997) Neuroblastoma in adults and adolescents: An indolent course with poor survival. Cancer 79(10): 2028-2035.

11. Kaye JA, Warhol MJ, Kretschmar C, Landsberg L, Frei III E (1986) Neuroblastoma in adults. Three case reports and a review of the literature. Cancer 58(5): 1149-1157.

12. Kushner BH, Kramer K, LaQuaglia MP, Modak S, Cheung KV (2003) Neuroblastoma in adolescents and adults: The Memorial Sloan-Kettering experience. Medical and Pediatric Oncology 41(6): 508-515. 